\title{
eJRIEPS
}

Ejournal de la recherche sur l'intervention en éducation physique et sport

Numéro spécial 1 | 2018

Comment penser les articulations entre approches

didactiques et ergonomiques pour étudier les

pratiques d'enseignement et de formation?

\section{La technologie de l'intervention éducative : une interface entre ergonomie et didactique}

Technological approach, technicities registers, facets, artifact, uncertain nature, emancipation

Serge Éloi, Gilles Uhlrich et Laurent Grillet

\section{(2) OpenEdition}

Journals

Édition électronique

URL : http://journals.openedition.org/ejrieps/342

DOI : 10.4000 /ejrieps.342

ISSN : 2105-0821

Éditeur

ELLIADD

\section{Référence électronique}

Serge Éloi, Gilles Uhlrich et Laurent Grillet, «La technologie de l'intervention éducative : une interface entre ergonomie et didactique », eJRIEPS [En ligne], Numéro spécial 1 | 2018, mis en ligne le 01 avril 2018, consulté le 01 août 2019. URL : http://journals.openedition.org/ejrieps/342 ; DOI : 10.4000/ ejrieps.342

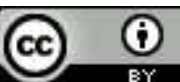

La revue eJRIEPS est mise à disposition selon les termes de la Creative Commons Attribution 4.0 International License. 
eJRIEPS Numéro Spécial n¹ Avril 2018

La technologie de l'intervention éducative :

une interface entre ergonomie et didactique

Serge Éloi ${ }^{*}$, Gilles Uhlrich ${ }^{\star *}$ et Laurent Grillet ${ }^{*}$

* Université Paris Est Créteil (UPEC), Laboratoire LIRTES, EA 7313, France

** Université Paris Sud, Université d'Orléans, Laboratoire CIAMS, EA 4532, France

\section{Résumé}

Dans le cadre d'un travail de réflexion concernant la caractérisation des recherches technologiques sur les PPSA ${ }^{2}$, il semble aujourd'hui indispensable de les situer au regard des travaux en ergonomie et en didactique afin d'en déterminer les périmètres respectifs. Nous sommes engagés depuis des années dans l'analyse de l'efficacité des activités de formation en sport proposées par différents acteurs. Cette position interroge, selon une logique ergonomique, les dispositifs de formation tels qu'ils sont proposés aux apprenants tout en s'intéressant à l'activité de l'intervenant qui le fait fonctionner. Mais cette opération ne se réalise que si l'intervenant dans une logique didactique, adapte la pratique par les contenus d'enseignement qu'il propose au regard des besoins des pratiquants. Par cette action, il exploite alors le caractère incertain des dispositifs de formation et accepte la nécessaire émancipation du pratiquant pour qu'il se développe. Nous montrons ici que la démarche technologique par la mobilisation articulée des concepts de registres de technicités, de facettes et d'artefacts permet au chercheur d'interroger cette activité singulière de l'intervenant au croisement de l'ergonomie et du didactique.

Mots clés: Approche technologique, registre de technicité, facettes, artefact, caractère incertain, émancipation.

\section{Introduction}

Quelle idée curieuse que de vouloir discuter de la pertinence d'un lien, a priori hypothétique, entre ergonomie et didactique. En quoi ces deux disciplines peuvent trouver un intérêt à se scruter, à se comparer, voire à partager ? Au premier abord, rien ne laisse envisager qu'une telle collaboration puisse aboutir à de quelconques résultats. Pourtant, ${ }^{2}$ Pratique Physique Artistique et Sportive 


\section{eJRIEPS Numéro Spécial n¹ Avril 2018}

en portant notre regard au-delà des lieux communs, il semble bien que ce rapprochement puisse bénéficier aux deux postures. Nous voulons montrer ici que la mise en perspective de cette confrontation peut être matérialisée au travers de la démarche technologique. Nous aborderons dans ce texte le cas spécifique des Pratiques Physiques Sportives et Artistiques (PPSA).

\section{L'ergonomie : quelle ergonomie ?}

La définition adoptée par l'Association Internationale d'Ergonomie (IEA) est la suivante :

"L'ergonomie (ou Human Factors) est la discipline scientifique qui vise la compréhension fondamentale des interactions entre les humains et les autres composantes d'un système, et la profession qui applique principes théoriques, données et méthodes en vue d'optimiser le bien-être des personnes et la performance globale des systèmes ". II ne s'agit pas ici de discuter du bien-fondé de cette définition mais de remarquer que l'ergonomie peut être en même temps une discipline scientifique et une profession. Cette particularité nous amène à pointer le caractère pragmatique du point de vue ergonomique.

1. 1. Ergonomie constructive - ergonomie progressiste ?

Certains spécialistes de la discipline apportent cependant quelques nuances à la définition de principe. Pour Falzon, le champ d'action de l'ergonomie doit s'étendre aux dimensions humaines qu'elles soient physiques, cognitives ou psychiques autant qu'à des secteurs tels que la conception, la production industrielle, les systèmes de santé, les services, les bureaux d'étude, etc. (Falzon, 2004). "Concevoir des situations de travail participant au développement professionnel est ainsi devenu un enjeu majeur et essentiel de l'ergonomie » (Falzon, 2013 ; cité par Villemain et Lémonie, 2014, p. 26). Vu sous cet angle, l'ergonomie ne se contenterait pas d'assurer la force de production au service d'un rendement du travail, "de prévenir les risques (accidents, maladies), de minimiser la fatigue (liée au métabolisme de l'organisme, à la sollicitation des muscles et des articulations, au traitement de l'information, à la vigilance), de créer les conditions d'un travail satisfaisant »(Falzon, 1996, p. 234). Elle contribuerait aussi à la santé du travailleur. La santé, c'est justement l'un des grands enjeux actuels des pratiques physiques et/ou sportives. Mais comme le pointe Falzon pour le champ d'action de l'ergonomie, l'appréhension de la santé dans le cadre des PPSA peut s'envisager selon deux prismes. Nous pouvons distinguer les prescriptions institutionnelles; la santé qui 


\section{eJRIEPS Numéro Spécial n¹ Avril 2018}

s'entretient par la pratique physique comme la condition de bon fonctionnement de l'individu dans son environnement professionnel. Dans cette perspective, les textes officiels de l'éducation physique et sportive dans le système scolaire invoquent la construction d'une " compétence générale " des élèves qui serait, "entretenir sa santé par une activité physique régulière " (BO $n^{\circ} 11$ du 26 novembre 2015, relatif au collège, cycle trois et quatre du collège). L'autre perspective plus ambitieuse qui considère la construction de la santé par une activité dans les PPSA comme un facteur de développement du pratiquant. Précisions ici quelques conséquences de cette caractérisation de la santé dans le cadre de l'ergonomie selon un double prisme.

1. 2. Santé et performance sont-elles compatibles?

Falzon défend l'hypothèse que santé et performance sont indissociables : "la santé est une condition de la performance et réciproquement la performance est une condition de la santé » (Falzon \& Mas, 2007, p. 732). II rejoint Clot (1999) qui évoque le «travail empêché " pour défendre l'idée selon laquelle la performance est une source de satisfaction, que le désir de "faire bien » est souvent entravé et que c'est cette entrave qui finit par générer de l'insatisfaction ou du stress. Clot aime à citer la formule de Canguilhem qui postule que : « Je me sens bien dans la mesure où je me sens capable de porter la responsabilité de mes actes, de porter des choses à l'existence et de créer entre les choses des rapports qui ne leur viendraient pas sans moi » (Canguilhem, 2002, p. 68).

\section{Le débat « santé et PPSA »}

Le concept de santé instrumentalisé ces dernières années par le ministère des sports (cf. loi Fourneyron) se focalise sur des visées utilitaires. On peut y repérer un discours idéologique de la santé sous-tendu par la volonté politique de réduire au plus juste les dépenses qui lui sont liées. On peut aussi y voir la volonté de maintenir l'outil de production à son niveau le plus efficient. Cette remarque peut sembler extrêmement polémique mais nous soutenons qu'elle a toute sa place dans notre débat épistémologique. Dans l'idéologie actuelle qui tend à justifier les actions ministérielles en faveur de la prescription de séances d'activités physiques par les médecins, deux argumentaires récurrents reviennent dans le discours politique :

- II faut se maintenir en bonne santé pour coûter le moins possible à la société (réduction du déficit de la sécurité sociale). 


\section{eJRIEPS Numéro Spécial n¹ Avril 2018}

- Il faut se maintenir en bonne forme pour pouvoir être productif (au-delà de la " maintenance » de l'outil humain de travail s'est développée une rhétorique relative à la lutte contre l'absentéisme).

Tout le monde pourrait s'en féliciter à la nuance importante qu'ici, la dimension culturelle du sport comme fait social est niée.

2. 1. Santé et PPSA : culpabilisation et prescription

II nous faut décrypter le discours politique qui justifie la mise en place des dispositifs qui voient le jour depuis quelques années. Nous rappelons que la Ministre Fourneyron a mis en place le «sport sur ordonnance ». Dans le cadre du parcours de soins des patients atteints d'une affection de longue durée, le médecin traitant peut prescrire une activité physique adaptée à la pathologie, aux capacités physiques et au risque médical du patient ${ }^{3}$.

Quelle lecture peut-on faire de ce type de mesure ?

2. 1. 1. De la culpabilisation...

Un discours se développe de plus en plus qui tend à expliquer que les problèmes qui nous affectent seraient dus à notre négligence. Nous fumons trop, nous mangeons mal, nous buvons sans modération, nous ne bougeons pas assez. Nous sommes nous-mêmes les coupables de tous nos maux.

Cependant, les statistiques ${ }^{4}$ sur les conditions de travail, même si elles se sont relativement améliorées depuis 50 ans, sont stupéfiantes. Cinq cents personnes meurent tous les ans au travail en France. Et si le nombre d'accidents du travail diminue pour les hommes $(-28,6 \%)$ il est en forte progression pour les femmes (+ $28 \%)$. On compte 624.625 accidents du travail pour l'année 2015. Dans le même temps, les maladies professionnelles reconnues ont augmenté de 110,4\% entre 2001 et 2015. Ici encore, ce sont les femmes qui sont les plus impactées (+ $155 \%)$. Malgré ces chiffres stupéfiants, les pouvoirs publics ne pointent le doigt que vers un seul coupable : les salariés.

Ce serait donc une mission de santé publique que de nous rééduquer.

Pourtant, nous passons au minimum 7 heures par jour au travail, auxquelles il faut ajouter le temps de transport (68 minutes en moyenne en région parisienne). À titre d'exemple,

${ }^{3}$ Décret $n^{\circ}$ 2016-1990 du 30 décembre 2016 relatif aux conditions de dispensation de l'activité physique adaptée prescrite par le médecin traitant à des patients atteints d'une affection de longue durée

${ }^{4}$ Statistiques 2015 de sinistralité au travail des salariés - Caisse Nationale de I'Assurance Maladie des Travailleurs Salariés (CNAMTS). 


\section{eJRIEPS Numéro Spécial n¹ Avril 2018}

une hôtesse de caisse dans un supermarché peut manipuler jusqu'à une tonne de produits par heure. Mais pour la maintenir en bonne forme, il lui faudrait pratiquer une activité sportive après sa journée de travail.

\section{1. 2. ...À la prescription}

Dans ce contexte, n'est-il pas injuste de montrer du doigt ceux que la maladie touche plus que d'autres ? À quel résultat cela peut-il aboutir sur ceux qui pâtissent de ces conditions de vie et subissent en plus une obligation qui ne font qu'alourdir leur quotidien ? Quel sens peut avoir une injonction ministérielle aux problèmes de santé ressentis par les malades?

Car la santé, ce n'est pas produire du mouvement. « Je me sens bien dans la mesure où je me sens capable de porter la responsabilité de mes actes... » (cf. ci-dessus).

Les pratiquants doivent donc se trouver en posture de porter la responsabilité de leurs actes en toute conscience et bien sûr, sans conséquence tragique pour leur intégrité physique ou mentale. Mais ces pratiques, pour susciter un investissement parfois important, doivent relever d'un enjeu accepté.

2. 2. Loin du discours idéologique : les PPSA comme facteur de développement Nous pensons que les PPSA sont un domaine où les prises de responsabilités sont « endossables» à la condition que les enjeux restent symboliques. Jeu l'avait bien compris lorsqu'il écrivait : «Création collective, instinctive, continue, dynamique grandiose de l'imaginaire, le sport traverse avec assurance l'histoire des peuples et n'a pas été inventé, au cours des âges, sur décision des princes ou recommandation des philosophes. II est vivant, populaire, spontané. II est émotion. II est passion » (Jeu, 1977, p. 9). Pour une erreur de décision, on évoque un mauvais choix tactique. Mais il peut être sans conséquence car au cours de la rencontre sportive, d'autres choix pourront être faits. C'est un lieu où la dimension fantasmagorique doit être préservée. On parle de mort symbolique en cas de défaite. On parle de résurrection quand l'équipe qui est menée au score retourne la situation in extremis à la fin du temps réglementaire. Ici encore la symbolique.

Caillois soulignait aussi que notre adhésion à telle pratique est sous-tendue par des attitudes humaines fondamentales. Dès 1958, il réalisait le projet de catégoriser les jeux : « je propose à cette fin une division en quatre rubriques principales selon que, dans les jeux considérés, prédomine le rôle de la compétition, du hasard, du simulacre ou du vertige. Je les appelle respectivement Agön, Alea, Mimicry et Ilinx » (Caillois, 1958/2009, 


\section{eJRIEPS Numéro Spécial n¹ Avril 2018}

p. 47). Cette classification renvoie à nos émotions, à nos ressentis et donc à un investissement volontaire dans ces pratiques.

Pratiquer, ce n'est donc pas s'agiter. C'est investir un espace social dans lequel chaque individu un rôle à jouer. Et c'est bien cette dimension métaphorique que l'intervenant doit faire valoir auprès des pratiquants. Encore faut-il que les disciplines proposées rendent crédibles les postures que les acteurs du jeu aiment à prendre. Ici, on ne peut pas faire semblant sous peine de l'apparition d'un désintérêt total et à terme d'un abandon de la pratique. Jeu relevait déjà en 1977 que «pour beaucoup cependant, tout doit servir à quelque chose et le sport effectivement dans une partie de ses attitudes n'échappe pas à l'obsession utilitariste... On a donc perdu de vue le caractère héroïque et sacré, la dimension symbolique de l'effort. On ne retient que l'intérêt pratique, militaire, médical, hygiénique, éducatif, que sais-je ? On fait du sport utile » (Jeu, 1977, p. 55).

La pratique ne peut perdurer que si le couple «sens - responsabilité » reste en mouvement et il apparaît bien que l'un des moteurs de ce mouvement soit l'enjeu. II ne peut y avoir responsabilité que s'il y a enjeu, que dans la mesure où le pratiquant se sent capable de porter la responsabilité de ses actes. Le sens de ses actions doit rester premier et par la même, la capacité de se construire des espaces d'initiatives et de liberté. C'est en recherchant cette posture du pratiquant que l'intervenant va donc interroger les PPSA.

\section{Le didactique et le problème des références dans l'enseignement des PPSA}

Dans le domaine des PPSA, contrairement à la chimie ou à la physique, il n'y a pas de savoir savant, pas d'équivalent du théorème de Pythagore ou du théorème de la poussée d'Archimède. Certes, certaines pratiques peuvent être éclairées par ces sciences d'appui mais il n'existe pas de théorème de la bonne passe, ou d'axiome du démarquage. Du coup dégager ce qui est du savoir «incontournable» et ce qui relève du plus « accessoire » est un véritable travail didactique qui n'est pas sans poser de problèmes lorsqu'il s'agit d'enclencher le processus de transposition didactique c'est-à-dire, le processus qui permet de passer d'un savoir savant en un savoir à enseigner (Chevallard, 1985).

3. 1. Les PPSA : des pratiques sociales avant tout

Pour trouver une issue à ce problème Martinand $(1981$; 1986) a proposé de parler de pratiques sociales de référence. La transposition didactique ne peut donc pas consister à 
eJRIEPS Numéro Spécial n¹ Avril 2018

transformer les savoir savants en savoir à enseigner. Il faut procéder à un travail de modélisation précédent toute transposition didactique dans le domaine des PPSA.

\section{1. 1. Qu'est-ce que modéliser?}

Modéliser, c'est produire des connaissances utiles à l'intervention (Martinand, 1987).

La démarche de modélisation se situe pleinement dans une perspective technologique car elle nécessite :

- de partir de la pratique pour retourner à la pratique

- de prendre en compte la complexité des activités réelles

- de produire de nouveaux concepts permettant de caractériser des situations typiques

- de permettre d'énoncer des règles d'efficacité.

Or, les PPSA sont des pratiques sociales.

Elles s'organisent au sein de structures sociales plus ou moins normalisées (clubs, comités, ligues, fédérations, fédérations internationales, ligues professionnelles...). Elles se sont développées au cours du temps constituant pour les secteurs de l'enseignement, de l'entraînement et de l'animation, des pratiques de référence. Dans certains secteurs des activités humaines, lorsqu'il y a absence de savoirs savants, les pratiques sociales constituent les références permettant de déterminer les savoirs à enseigner.

\section{1. 2. Non pas une, mais des références}

Se pose alors la question de la référence car ces pratiques sociales ne sont pas monolithiques. Elles peuvent varier en fonction des lieux où elles se déroulent, des pratiquants qui s'en emparent et de leur expression plus ou moins « sauvage » (hors cadre officiel). Le basket en est un bel exemple puisque l'on peut dénombrer au moins 3 pratiques sociales distinctes. On peut faire référence au basket fédéral qui s'appuie sur un règlement européen. Le Basket Américain, très visible sur les chaînes sportives, a ses propres règles et constitue une autre forme de pratique. Cela nécessite donc pour chaque instance représentative de ses deux formes de jeu de se mettre d'accord lorsque des compétions mondiales doivent être organisées (championnat du monde, jeux olympiques) car la compétition doit se dérouler avec un règlement commun. Et puis, il y a le basket des «playgrounds» qui se joue dans les quartiers, au pied des immeubles. Cette multiplicité des pratiques peut être également relevée pour le volley-ball où des contextes très différents peuvent être observés. Chacun connaît le volley-ball «traditionnel » qui se pratique en intérieur et en 6 contre 6 . Mais le beach-volley qui se joue sur la plage en 2 contre 2 est aussi au programme des jeux olympiques. II existe par ailleurs un 


\section{eJRIEPS Numéro Spécial nº1 Avril 2018}

championnat de France officiel de volley de plage en 3 contre 3. On peut aussi évoquer les tournois estivaux où les amateurs s'affrontent en 3 contre 3 ou en 4 contre 4 tout le long du littoral voire, sur le champ de Mars, à Paris. De la même façon, on peut relever que le rugby peut se jouer à 15 , à 13 ou à 7 . Et que dire de la danse ?

\section{2. La transposition didactique : un processus en question}

II n'existe donc pas une pratique sociale de référence mais des pratiques sociales de référence. Comment choisir alors telle forme de pratique plutôt que telle autre et pour quel contexte de l'intervention éducative (enseignement, entraînement, animation) ?

3. 2. 1. Les problématiques liées à la transposition didactique

\section{- Rapport entre pratiques familières aux apprenants et pratiques de référence.}

L'un des arguments qui préside à ce choix s'appuie sur le fait que certaines formes de pratiques sont plus adéquates pour faire entrer les élèves dans les logiques de ces disciplines sportives ou artistiques. On peut imaginer que les élèves d'un quartier sensible pourraient être plus enclins à entrer dans la danse par le hip hop plutôt que par la danse classique.

\section{- Écart entre technicité dans la référence choisie et contenus des disciplines scolaires.}

Le problème soulevé ici est celui de la légitimité de la référence, c'est à dire de son adéquation avec les représentations des pratiquants à qui l'on dit qu'ils vont faire du basket, du rugby ou de la danse. Martinand souligne que « le problème pour le didacticien est celui de l'authenticité par rapport à cette pratique »(Martinand, 1981, p. 154). Comment transposer les pratiques sans les dénaturer? Est-il primordial de viser l'enseignement des technicités les plus évoluées qui sont souvent celles du plus haut niveau?

\section{- Relation entre sens des pratiques et sens des apprentissages.}

Cela nous renvoie à ce qui fait sens chez le sujet qui s'investit dans ces pratiques. Ici encore, il s'agit de jouer sa vie sans risquer de la perdre. L'intervenant doit alors préserver des enjeux subjectifs forts dont les conséquences objectives sur l'intégrité physique et mentale des pratiquants seront sans effet. Le seul effet recherché ici est celui qui va provoquer l'adhésion des pratiquants à la pratique de référence choisie, la possibilité de faire le premiers pas, quitte à évoluer ensuite vers une autre pratique, une autre référence. 


\section{eJRIEPS Numéro Spécial n¹ Avril 2018}

Toutes ces problématiques renvoient en fin de compte à la question de la transmission. Le problème du didacticien peut se résumer ainsi: Qu'est ce qui est digne d'être enseigner ? Qu'est-ce qui a une valeur culturelle ? Que doit-on transmettre ?

C'est-à-dire :

- qu'est-ce que l'enseignement public se doit de transmettre aux citoyens de demain ?

- Qu'est-ce que les futurs citoyens doivent appréhender du secteur des PPSA pour être des contemporains actifs?

\section{2. 2. Modélisation et essence}

Une voie de réponse tient dans ce que nous avons appelé l'essence des pratiques (Éloi \& Uhlrich, 2001). L'effort de modélisation nécessaire en vue d'une transposition didactique maîtrisée doit mettre à jour l'essence de ces pratiques, leurs rapports, c'est-à-dire, au sens du matérialisme dialectique, une " relation entre des termes essentiellement déterminés l'un par l'autre et par l'unité qu'ils constituent "(Sève, 1980, p. 704). Ici débute le travail de modélisation qui se doit d'expliciter les relations dialectiques qui organisent les éléments qui constituent les pratiques. Par exemple, les sports collectifs ne devraient être envisagés que par la prise en compte du rapport attaque-défense. Pour sa part, l'escalade devrait être appréhendée au travers de son rapport risque-sécurité. La transposition didactique est donc au cœur de nombreuses problématiques. Elle relève de la non-linéarité. II n'y a pas d'automatisme entre enseignement et apprentissage. On ne peut donc faire fi de la complexité inhérente à la transmission-appropriation des savoirs. Le contexte de l'enseignement-apprentissage doit alors être envisagé comme un système complexe.

\section{2. 3. La complexité inhérente au didactique}

La multiplicité des composants qui interagissent dans une action de transposition didactique c'est-à-dire, ce qui est relatif aux contenus, aux intervenants et aux pratiquants d'une part, mais aussi, à la diversité des interrelations qui lient ces composants d'autre part, ne sont que quelques-unes des caractéristiques plus ou moins visible de la «didactique du volley-ball » ou de la «didactique de la danse ». Rien n'est écrit pour toujours et rien n'est immuable dans ces systèmes complexes car une autre dimension déterminante de la complexité est l'imprévisibilité potentielle des diverses conduites au sein du système. "La notion de complexité implique celle d'imprévisible possible, d'émergence plausible du nouveau et du sens au sein du phénomène que l'on tient pour complexe »(Le Moigne, 1999, p. 3). Une modélisation est donc un système vivant qui 


\section{eJRIEPS Numéro Spécial n¹ Avril 2018}

nécessite des remises à jour perpétuelles et qui est susceptible de produire « une génération spontanée de propriétés du tout qui n'appartiennent en rien aux parties alors même que l'on ne veut le penser que comme étant le tout-des-parties » (Sève, 2005, p. 126). C'est ce paradoxe que l'on appelle l'émergence. Mais la plupart du temps, l'émergence est vue comme l'apparition magique d'une qualité nouvelle que l'on ne sait expliquer. C'est l'une des spécialités des tenants des théories de l'action située que de nous dire que tout vient au sujet, le monde lui apparaît, la solution émerge. II y a là un abus de langage, une sorte de mystification qui amène le chercheur à ne se questionner que sur les choses, là où les solutions se trouvent dans le rapport des choses entre elles. Comme le souligne Sève : « en tout domaine, le niveau le plus profond est de l'ordre non de la chose mais du rapport » (Sève, 2005, p. 129). II nous faudra donc suivre le précepte de Boileau, «cent fois sur le métier remettez votre ouvrage » pour comprendre ce qui émerge et d'où cela émerge en fonction des secteurs d'intervention, des disciplines sportives abordées ou des publics concernés.

\section{2. 4. Ergonomie et didactique : quel lien ?}

À ce stade de notre exposé, il nous paraît nécessaire de synthétiser l'argumentaire exposé précédemment qui s'appuie sur des concepts et définitions d'auteurs des champs de l'ergonomie et de la didactique. Pour notre part, l'ergonomie doit se préoccuper d'analyser le facteur humain dans des activités où des opérateurs sont au cœur du dispositif de production au sens large (matériel, de service, culturel, etc.). Cette préoccupation nécessite de recouper les dimensions physiques, cognitives et psychiques dans des secteurs aussi variés que la conception, la production, les services, les systèmes de santé, l'intervention éducative, les pratiques sportives, etc. Plus encore, l'ergonomie se fixe comme objectif d'analyser les savoirs, de les améliorer, de participer au confort des humains engagés dans leurs activités de production, de concevoir des situations de travail participant à leur développement professionnel, de favoriser leur performance et dans le même temps, de s'assurer de leur état de santé.

De son côté, la didactique se doit de participer au travail de modélisation des pratiques sociales. Elle doit décider des contenus à transmettre (philosophie, politique, éthique, etc.), construire les curriculums de formation (programmes scolaires), organiser les dispositifs (prescriptions, critères d'évaluation, contrat didactique, etc.), donner du sens à l'engagement des pratiquants quels que soient les secteurs de pratiques (enseignement, entraînement, animation) en reliant les contenus d'enseignement aux pratiques culturelles qui les légitiment. 
eJRIEPS Numéro Spécial n¹ Avril 2018

Ergonomie et didactique semble donc situées sur des planètes différentes. L'ergonomie se doit de transformer les conditions concrètes de l'exercice du métier. La didactique a pour objet de mettre en adéquation le système de formation et la population concernée. L'articulation ergonomie-didactique semble donc peu probable sauf à considérer que ce sont les problèmes de mise en action des solutions envisagées, les mises en œuvre permettant de passer du «pensé » au «pragmatique » qui peuvent former une passerelle entre les deux disciplines. C'est ici que la technologie intervient pour jouer ce rôle de passerelle.

\section{La démarche technologique : un pont jeté entre ergonomie et didactique}

La démarche technologique est motivée par le désir de résoudre des problèmes. Elle s'attache à produire des connaissances pragmatiques en interpellant les modèles théoriques.

Pour Staudenmaier (1988), le savoir technologique est un style de connaissances propre et particulier intégrant des concepts scientifiques, des données spécifiques, des théories distinctes des sciences et du savoir-faire. Dans le domaine des STAPS, cette posture a permis d'associer à des concepts scientifiques clefs (moment d'inertie, accélération, forces, temps de réaction, etc.) des données casuelles (apparitions d'évènements inattendus, manipulations d'informations, mises en œuvre de combinaisons de jeu, etc.) et des habiletés techniques (qui relèvent du domaine de la création technique). La technologie dont les situations typiques relèvent d'une démarche pragmatique n'occulte pourtant pas les concepts scientifiques. Elle s'en empare pour proposer des solutions. Elle les interpelle lorsque ce qui est réalisé ne trouve pas encore d'explication scientifique. La technologie prend en considération les problèmes de terrain qui constituent en même temps, le point de départ de la démarche et les obstacles qu'il faudra franchir, soit en mobilisant les connaissances scientifiques, soit en mobilisant du savoir-faire, de l'ingéniosité ou de la malice. La démarche technologique ne peut se développer que dans le respect de la complexité inhérente aux activités humaines car le terrain reste son juge de paix.

La démarche technologique se concrétise en cours de recherche par un affinement et une évolution de ses outils (méthodologie, dispositif, artéfacts...) et donc par une succession d'essais techniques qui permettent d'expérimenter, d'affiner puis de concrétiser les solutions à mettre en œuvre. Cette dynamique amène le pratiquant à vivre des situations qui ont du sens et offrent des espaces d'initiatives, l'intervenant étudie les 
eJRIEPS Numéro Spécial n¹ Avril 2018

PPSA dans le but d'adapter ses dispositifs et ses contenus, le chercheur interroge la pertinence du dispositif au regard de l'activité déployée par l'intervenant et les pratiquants. Pour toutes ses raisons, la démarche technologique semble être en position de faire le lien entre ergonomie et didactique.

\section{1. Soubassements théoriques}

Dans le cadre de recherches technologiques, nous travaillons en nous inspirant de la méthode instrumentale en psychologie de Vygotsky (1931/2014). Pour lui, il existe des « outils » ou « instruments » psychologiques qui organisent des « techniques intérieures » (ex : routines de réflexions, outils mnémotechniques, schémas tactiques). II considère alors que ces instruments psychologiques sont pour l'individu des constructions artificielles orientées vers la maitrise de son travail (ex : le langage, la numération, les schémas). Dans le processus comportemental du formé, l'instrument transforme le cours de l'activité en déterminant la forme de son travail. II crée artificiellement les conditions d'une modification de fait des «modes de faire». Les comportements sont alors « d'essence artificielle ». Ils ne sont pas innés et n'émergent pas sans raison. Ils sont ancrés dans une réalité historique et sociale. C'est en ce sens que Vygotski évoque le développement d'un individu dans une perspective historico-culturelle.

4. 2. La démarche technologique : un projet d'intervention

Cette prise en compte de la méthode instrumentale en psychologie amène à penser les conditions pour apprendre. L'intervenant éducatif a pour mission de créer des contextes artificiels qui permettent aux sujets de percevoir les motifs de leur action, de repérer les éléments les plus significatifs de la situation et de se construire des instruments psychologiques. Pour le chercheur, C'est dans cette dynamique que la recherche technologique se place. Elle interroge l'efficacité des dispositifs mis en place dans les PPSA par les intervenants éducatifs au regard des apprentissages réalisés par les élèves. La technologie est donc envisagée comme un « usage rationnel des techniques dans le cadre de la conception, la mise en œuvre et l'évaluation d'un projet d'intervention sur le réel. » (Bouthier, 2005). Dans la dynamique des PPSA, la démarche technologique structure les projets d'intervention quel que soit leur secteur d'application (enseignement, entrainement, animation). Se pose alors la question générale de l'interrogation de la formation des techniques. Nous avons ces dernières années (Éloi \& Uhlrich, 2011 ; 2013 ; 2014 ; Uhlrich \& Éloi, 2016) présenté une perspective qui vise à dépasser une vision techno-centrée de la technique. Il convient d'abandonner l'idée qu'une simple mobilisation de la bonne forme corporelle permettrait, par une application mécanique, de résoudre les 


\section{eJRIEPS Numéro Spécial n¹ Avril 2018}

problèmes complexes portés par les PPSA. Nous mobilisons le concept de technicité (Combarnous, 1984) qui lie habileté corporelle et motif d'agir du pratiquant. Nous y associons la notion de registre de technicité (Éloi \& Uhlrich, 2011) qui vient caractériser des éléments techniques en les regroupant autour de modes de fonctionnement. " La notion de technicité et ses composantes apparaissent aujourd'hui insuffisantes pour penser la construction et l'évolution des disciplines. C'est pourquoi dans la dernière période, à la fois pour la didactique des activités physiques et sportives et pour la didactique des sciences et de la technologie, j'ai proposé la notion de registres de technicité " (Martinand, 1994, p. 69). Ainsi, le technologue n'envisage pas le geste sportif dans son acception simpliste, il pense la technicité au filtre de registres.

4. 3. Registres de technicité dans les PPSA et points de vue des sujets Dans le domaine des PPSA, la technicité peut s'exprimer selon différents registres. Nous avons distingué (Uhlrich, Éloi \& Bouthier, 2011) le registre de maîtrise qui correspond aux techniques mobilisées, le registre de lecture qui correspond à la capacité d'interpréter ce qui se déroule dans la pratique, le registre de transformation qui porte davantage sur la prise en compte d'évolution ou d'innovation réalisées dans la pratique et enfin le registre de participation qui envisage de s'engager dans tous les autres rôles qui font la pratique (arbitre, juge, entraîneur, etc.).

Cette énumération (non exhaustive) des registres ne prend toute sa valeur que lorsqu'on l'envisage comme un système articulé. Cette vision organisée des techniques s'accompagne d'un autre filtre d'analyse. Les registres sont à interroger du point de vue du pratiquant lorsqu'il est engagé dans l'action, mais aussi du point de vue de l'intervenant éducatif qui fait fonctionner des dispositifs dans lesquels sont plongés les pratiquants. II semble également nécessaire de prendre en compte le point de vue du formateur de formateur qui met en place des dispositifs qui orientent les futurs intervenants éducatifs dans l'élaboration de situations didactiques. Et se décalant de l'ensemble de ces positions, il reste à envisager le point de vue du chercheur qui va interroger chacun des points de vue précédents ou les interactions entre les uns et les autres. Chaque registre peut être envisagé selon ces différents points de vue. Au lieu de " découper » la complexité de l'action sportive, le technologue envisage en les croisant, prise en considération d'un registre et prise en compte d'un point de vue. Mais ce n"est pas le seul filtre d'analyse de l'activité du couple intervenant-pratiquant que le technologue peut mobiliser. II interroge aussi ce que l'apprenant construit en tenant 


\section{eJRIEPS Numéro Spécial n¹ Avril 2018}

compte de ce que l'intervenant vise. Le chercheur analyse alors les facettes du pratiquant qui sont mises à contributions.

\section{4. Les facettes de l'action sportive}

Bouthier, depuis plusieurs années (Bouthier, 1984, 1988,1993) défend l'idée que tout individu engagé dans les pratiques sportives, mobilise des «facettes » de sa personne. Sans prétendre à l'exhaustivité, il précise que le sportif engage lors de ses réalisations son potentiel athlétique, focalise sa vigilance afin de prendre les décisions les plus pertinentes tout en tentant de se maitriser émotionnellement. II parle de facettes car il envisage la métaphore de « la boule à facettes » qui est éclairée par un projecteur. Ce « coup de projecteur » qui éclaire « la boule à facette », c'est celui des valeurs et des motifs d'agir du sujet qui s'engage dans l'action. Cet éclairage montre que l'individu va toujours privilégier une des facettes dans sa réalisation. On dit souvent d'un jeune joueur de rugby qu'il sait « lire le jeu » ou d'un tennisman qu'il est « stable émotionnellement », etc. En changeant de point de vue, l'intervenant éducatif va lui aussi cibler la facette prioritaire par laquelle il va tenter de faire progresser ses pratiquants. Par exemple, l'enseignant sait que lorsqu'il engage des débutants dans la pratique du rugby, il va lui falloir tenir compte principalement de la dimension émotionnelle vécue par les sujets. L'unité-totalité-complexité des différentes facettes de l'action sportive mobilisées à la fois en tension et en synergie constituent le gisement des savoirs et des pouvoirs de l'agir des PPSA. C'est un objet technique culturel. Or, c'est une représentation dynamique des facettes en interaction qui s'épaulent et s'influencent mutuellement qu'il faut envisager.

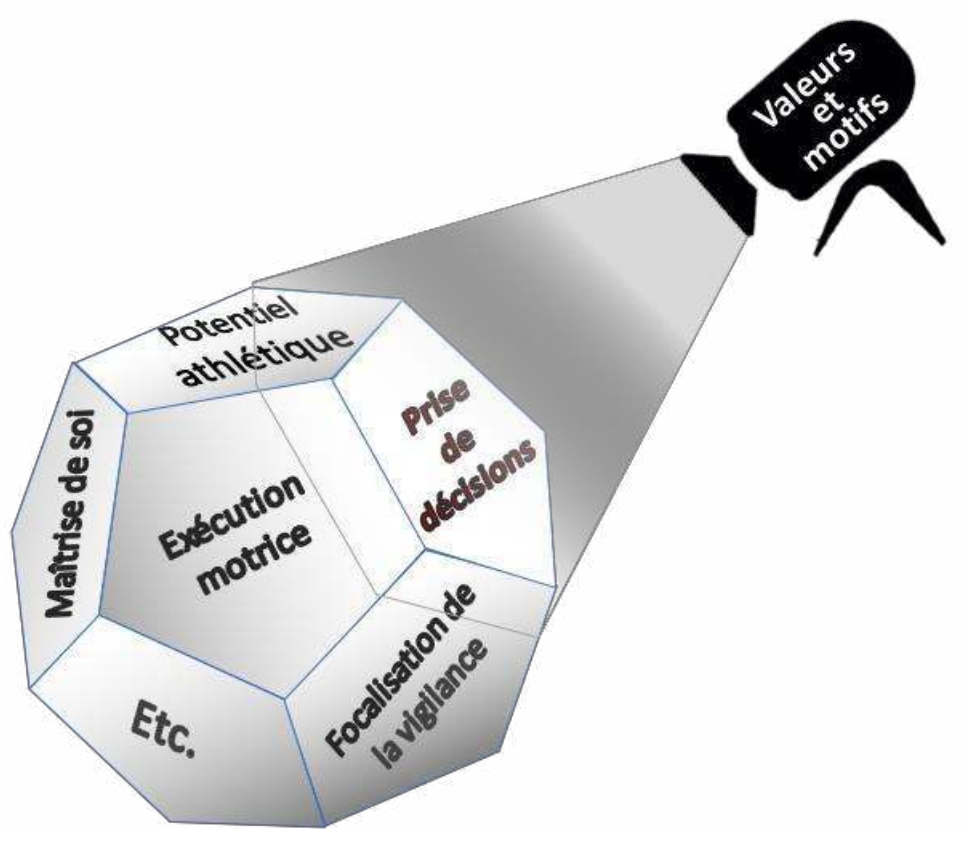


Figure 2. Les facettes de l'action sportive à partir des propositions de Bouthier (2014).

\section{La démarche technologique en STAPS}

Cette démarche tient en quatre points : une visée, une méthode, des connaissances et des propositions.

5. 1. Une visée

En récupérant des données sur le terrain dans la situation de l'action sportive, l'objectif est toujours de créer les conditions d'une proposition utile pour les pratiquants ou les intervenants. II s'agit donc de ne pas seulement en rester au plan des conclusions scientifiques mais bien d'envisager des dispositifs et de modes d'agir dont la recherche technologique a prouvé sa pertinence sans standardiser les résultats qu'ils peuvent générés. Dans cette logique, les recherches technologiques prennent inévitablement en compte la complexité de l'action sportive. L'une des principales conséquences de cette perspective est qu'il faut accepter qu'il n'y ait pas de réponse définitive, ce qui peut perturber le chercheur.

5. 2. Une méthode : Alimentation des registres de technicités par l'usage d'artéfacts La démarche technologique cherche à valider des résultats par la proposition d'essais techniques. L'étude se construit en fonction de la récupération des données utiles pour l'intervention. Pour catégoriser les attendus des recherches technologiques sur les PPSA, nous utilisons le filtre discriminant des registres de technicité (maîtrise, lecture, transformation ou participation) dans leurs interactions. La nécessité de caractériser les travaux amène à spécifier un registre principal et un ou plusieurs registres associés (Éloi, Uhlrich, 2014). Ainsi, le chercheur qui s'intéresse à la mobilisation des techniques du pratiquant en volley-ball va dans le dispositif mis en place, investiguer le registre de maitrise des joueurs. Pour autant, il ne peut dissocier cet aspect principal de son registre de lecture. Penser la technicité, c'est aussi prendre en compte la lecture des trajectoires de balle. La perspective technologique envisage le fonctionnement du sujet (pratiquants, intervenant, formateur de formateur, chercheur) dans sa relation aux « outils techniques » qui l'entourent. Dans le cadre des PPSA, nous pouvons distinguer des outils d'aménagement du milieu (les plots, les couloirs ou lignes d'eau en natation, etc.). Ils peuvent aussi prendre la fonction d'aide à la réalisation (le plan incliné en gymnastique, les planches en natation). Ils sont encore des outils d'observation (la grille d'observation, la vidéo, le logiciel statistique, etc.). Nous avons depuis de nombreuses années dépassés 


\section{eJRIEPS Numéro Spécial n¹ Avril 2018}

cette représentation figée de ce que sont les outils techniques pour envisager ces éléments comme des artefacts (Éloi \& Uhlrich, 2011 ; 2014 ; Uhlrich, Éloi \& Bouthier 2011 ; Uhlrich, 2005). Cette distinction marque volontairement le passage d'une vision techno-centrée à une vision anthropo-centrée. Dans cette dynamique, nous caractérisons l'artefact comme un incident volontairement introduit dans l'activité du sujet en vue de provoquer son activité et de développer ses compétences. La relation entre l'artefact et l'humain est interrogée au prisme de la genèse instrumentale (Rabardel, 1995). Nous interrogeons alors l'influence qu'exerce l'artefact sur l'activité du sujet mais en retour, nous prenons en considération ce que réalise le sujet pour transformer l'artefact. Ce rapport à l'artefact vaut pour le pratiquant comme pour l'intervenant. La spécificité technologique associe ainsi un aspect ergonomique (prise en main ou mise à sa main de l'artefact) qui met en évidence le didactique. La démarche technologique adopte le chemin inverse lorsque les contenus à proposer interrogent l'efficacité des dispositifs.

5. 3. Des connaissances construites à l'aide des dispositifs

Les recherches technologiques visent à élargir les procédures d'action des pratiquants et des intervenants éducatifs en envisageant une évolution des techniques. C'est donc en tenant compte des points de vue des acteurs (pratiquant, intervenant éducatif ou formateurs de formateur) voire dans leurs interactions que nous envisageons ces connaissances. Ainsi, nous avons pu montrer que dans le cadre de l'observation d'un match de rugby à 7 , un futur intervenant éducatif construit sa technicité de lecture en décrivant les évolutions des états d'équilibre et de déséquilibre entre l'attaque et la défense (Uhlrich \& Éloi, 2016). Nous faisons l'hypothèse que le futur intervenant éducatif capable de mieux lire le jeu des pratiquants, alimente en retour sa capacité à apprécier les situations lorsqu'il reprend lui-même le point de vue du pratiquant. Cette problématique qui suggère que la formation à la lecture du jeu (point de vue de l'observateur) pourrait se réinvestir dans le jeu (point de vue du joueur) doit donner corps à de nouvelles recherches.

5. 4. Des propositions pour la diffusion à la communauté professionnelle La démarche technologique vise à alimenter la communauté professionnelle. Les solutions au problème de départ ont pour vocation à être partagées et diffusées. Cela se concrétise par la production :

- d'outils (schémas, matériels spécifiques, logiciels informatiques, etc.)

- de dispositifs (organisation d'une simulation, formalisation d'une situation, etc.) 
eJRIEPS Numéro Spécial n¹ Avril 2018

- d'éléments plus conceptuels (modélisation des pratiques, guides de construction ou d'utilisation)

L'ensemble des éléments produit sont alors à disposition de tous les acteurs de l'intervention éducative et peuvent être éprouvés dans différents contextes afin de tester leur validité. C'est dans cette dynamique que nous nous sommes saisis du concept d'artefacts (Rabardel, 1995; Uhlrich, Éloi, 2011). Dans le cadre de l'intervention éducative, « L'incident artefactuel peut donc être caractérisé comme l'utilisation d'un objet matériel ou non, volontairement introduit par l'intervenant dans l'activité du sujet visant à la modification du cours normal de sa pratique dans le but de provoquer une compréhension nouvelle de son activité »(ibid., p.30). L'intervenant éducatif doit donc être en mesure de mobiliser voire de reconstruire de tels procédés.

\section{Les artefacts}

" La notion d'artefact désigne en anthropologie toute chose ayant subi une transformation, même minime, d'origine humaine » (Rabardel, 1995, p. 59). L'artefact est un objet technique qui peut prendre une forme matérielle et/ou conceptuelle. Pour nous, l'artefact constitue un obstacle qu'il s'agit de surmonter. C'est le pouvoir acquis pour dépasser les difficultés rencontrées qui permet de solutionner une nouvelle classe de problèmes. Cependant, dans le même temps il crée des conditions propices à la réalisation de l'action. Décrire le jeu de rugby en comptabilisant le nombre de touches de mêlée et de placage ne correspond pas à la même appréhension de la pratique que lorsqu'on observe les états d'équilibre et de déséquilibre entre l'attaque et la défense. L'intervenant éducatif qui sait pourquoi il propose un artefact à ses formés doit s'attendre à des effets multiples et étalés dans le temps. II reçoit les propositions des formés en acceptant un rapport nouveau à une temporalité différente des réponses tout comme à l'hétérogénéité des formes de réponses proposées. En ce sens la proposition d'artefacts répond à la prise en compte de la singularité des formés. Bien que l'artefact soit singulier, il est susceptible de provoquer des réponses différentes. Ce sont d'ailleurs ces différences qui sont porteuses de significations pour le technologue. Finalement, un artefact proposé par un intervenant sans qu'il prenne en compte cette multiplicité des réponses des apprenants est un objet d'intervention inerte. C'est sa position privilégiée au sein du système qui organise facettes et registres qui peut donner du sens et développer l'efficience du pratiquant. L'intrication des registres, des artefacts et des facettes forment le système instrumental de l'activité du pratiquant (figure 3). 


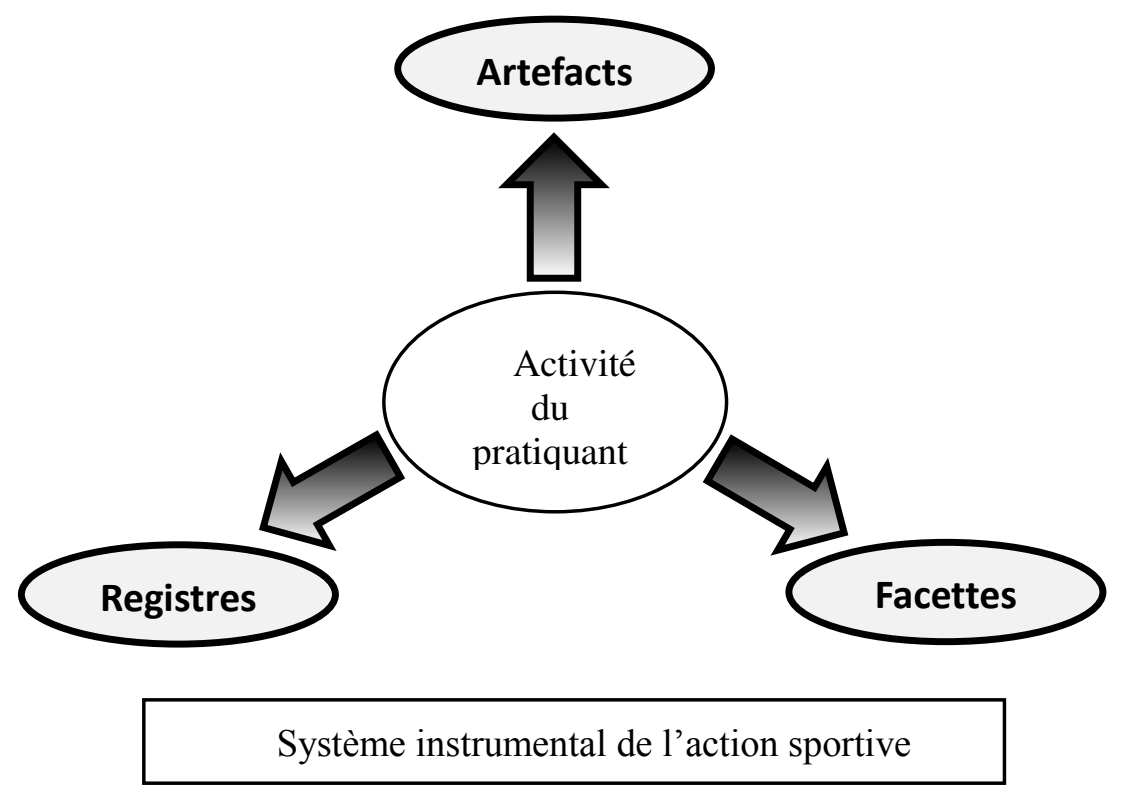

Figure 3. Le système instrumental de l'activité du pratiquant sportif.

\section{Conclusion : vers un système ergo-techno-didactique}

L'ensemble de ces réflexions nous amène à envisager un système qui articule les points de vue ergonomiques, didactiques et technologique selon deux modalités :

- celle de la compréhension fondamentale des interactions entre les humains et les autres composantes d'un dispositif de production (industriel, culturel, social).

- celle de la prise en compte des dimensions humaines qu'elles soient physiques, cognitives ou psychiques lors de la mise en œuvre de ces dispositifs.

Le système ergo-techno-didactique, pour fonctionner devrait mettre en synergie :

- la conception des situations de travail (situation d'enseignement/apprentissage ici)

- la santé comme une condition de la performance voire bien au-delà comme un facteur de développement de l'individu.

- la capacité du sujet à porter la responsabilité de ses actes par la mobilisation d'artefacts dans le cadre d'un processus de genèse instrumentale.

La représentation des trois approches pourrait se concrétiser par une " articulation 》 de leurs spécificités. Dans ces conditions, la technologie qui mobilise ces dispositifs en les mettant en œuvre selon trois modalités (registres de technicité, artefacts et facettes) se positionne comme une interface idéale pouvant permettre de faire le lien entre l'ergonomique et le didactique. 


\section{eJRIEPS Numéro Spécial n¹ Avril 2018}

\section{Bibliographie}

Bouthier, D. (1984). Sports collectifs : contribution à l'analyse de l'activité et éléments pour une formation tactique essentielle. L'exemple du rugby. Mémoire INSEP. Paris.

Bouthier, D. (1988). Les conditions cognitives de la formation des actions sportives collectives. Thèse de doctorat en psychologie. Université Descartes. Paris V.

Bouthier, D. (1993). L'approche technologique en STAPS : représentations et actions en didactique des APS. Diplôme d'habilitation à diriger des recherches, spécialité STAPS. Université Paris XI, Orsay.

Bouthier, D. (2005). Analyse de l'Activité en APS et développement des Compétences en Didactique professionnelle. Séminaire ISA-IDEES.

Bouthier, D. (2014). Iniciacion y perfeccionamento en los deportes colectivos : desarrollo de la pertinencia de la toma de decisiones en el juego en relacion con otros elementos de la accion. . In V. Lopez et J. Sagartal (eds): El aprendizaje de la accion tactica. Universitat de Girona, Diversitas 76, 99-132.

Caillois, R. P. (1958). Les Jeux et les hommes. Paris. Folio.

Canguilhem, G. (2002). Écrits sur la médecine, Seuil, Paris.

Chevallard, Y. (1985). La transposition didactique : du savoir savant au savoir enseigné. Recherche en didactique des mathématiques. La pensée sauvage éditions.

Clot, Y. (1999). La fonction psychologique du travail. Paris : PUF.

Combarnous, (1984). Les techniques et la technicité, Messidor/Éditions sociales.

Éloi, S. \& Uhlrich, G. (2001). Contribution à la caractérisation des sports collectifs : les exemples du volley-ball et du rugby. STAPS, 56, 109-125.

Éloi, S. \& Uhlrich, G. (2011). La démarche technologique en STAPS : analyse conceptuelle et mise en perspective pour les sports collectifs, eJRIEPS, 23, 2045.

Éloi, S. \& Uhlrich, G. (2013). La mobilisation d'un artefact réglementaire dans le cadre de la formation d'étudiants en STAPS. Une illustration en volley-ball. Recherche et formation, 21, 73-88.

Éloi, S. \& Uhlrich, G. (2014). Utilisation d'artefacts dans le domaine des pratiques physiques, sportives et artistiques (PPSA). Transformation, 12, 117-135.

Falzon, P. (1996) Des objectifs de l'ergonomie. In F. Daniellou (Ed.) L'ergonomie en quête de ses principes. Débats épistémologiques, 233-242. Toulouse : Octarès. 
eJRIEPS Numéro Spécial n¹ Avril 2018

Falzon, P. (2004). Ergonomie. Paris : PUF.

Falzon, P. (2013). Pour une ergonomie constructive. Ss le dir. de P. Falzon, Ergonomie constructive. Paris : PUF. 1-16.

Falzon, P. \& Mas, L. (2007) Les objectifs de l'ergonomie et les objectifs des ergonomes. In M. Zouinar, G. Valléry \& M.-C. Le Port (sous la coord. de), Ergonomie des produits et des services, XXXXII ${ }^{\circ}$ congrès de la SELF, Toulouse : Octarès ou http://ergonomie-self.org/wp-content/uploads/2016/01/actes-42-congres-self2007-saint-malo.pdf

Jeu, B. (1977/1983). Le sport, l'émotion, l'espace. Paris. Vigot.

Le Moigne, J.L. (1999). La modélisation des systèmes complexes. Paris : Dunod (2e éd).

Martinand, J.L. (1981). Pratiques sociales de référence et compétences techniques. À propos d'un projet d'initiation aux techniques de fabrication mécanique en classe de quatrième, in A. Giordan (coord.). Diffusion et appropriation du savoir scientifique : enseignement et vulgarisation. Acte des Troisièmes Journées Internationales sur l'Éducation Scientifique. (Pp. 149-154) Paris : Université Paris 7

Martinand, J.L. (1986). Connaître et transformer la matière. Éd. Peter Lang.

Martinand, J.L. (1987). Quelques remarques sur la didactique des disciplines. Les Sciences de l'Éducation, 2, 23-29.

Martinand, J.L. (1994). La didactique des sciences et de la technologie et la formation des enseignants. ASTER, 19, 61-75.

Rabardel, P. (1995). Les hommes et les technologies. Approche cognitive des instruments contemporains. Paris : Armand Colin.

Sève, L. (1980). Une introduction à la philosophie marxiste. Terrains/Éditions Sociales.

Sève, L. (2005). Émergence, complexité et dialectique. Odile Jacob.

Staudenmaier, J.M. (1988). L'histoire des sciences et la question : "les technologies sont-elles des sciences appliquées ? ". Courrier du Cethès, construire une éthique de l'enseignement scientifique, 5, 27-43.

Uhlrich, G. (2005). Rôle des artefacts matériels et cognitifs dans le développement des compétences de l'intervenant éducatif en rugby. Thèse de doctorat STAPS, Université de Bordeaux 2, non publiée. 
eJRIEPS Numéro Spécial n¹ Avril 2018

Uhlrich, G., Éloi, S. \& Bouthier, D. (2011). La technologie dans le contexte des STAPS : de la conception d'outils à la conceptualisation... et réciproquement. eJRIEPS, 23, 4-19.

Uhlrich, G. \& Éloi, S. (2016). Développer l'intelligence tactique en jeu par la pédagogie des modèles de décision dans la perspective francophone. eJRIEPS, 38, 38 - 62

Villemain, A., \& Lémonie, Y. (2014). Environnement capacitant et engagement des opérateurs : une mise en débat à partir de l'activité des techniciens de la base polaire Dumont d'Urville. Activités, 11(2), 26-43. http://www.activites.org/v11n2/v11n2.pdf.

Vygotsky, L. (1931/2014). Histoire du développement des fonctions psychiques supérieures. La Dispute. 